\title{
Towards Responsive Regulation
}

\author{
Introduction
}

\author{
Catriona McMillan
}

This section of the volume offers a contemporary selection of examples of where existing models of law and regulation are pushed to their limits. Novel challenges are arising that require reflection on appropriate and adaptive regulatory responses, especially where ethical concerns raise questions about the acceptability of the research itself. The focus in this section is on how these examples create disturbances within regulatory approaches and paradigms, and how these remain a stubborn problem if extant approaches are left untouched. The reference to 'responsive' here highlights the temporally limited nature of law and regulation, and the reflexivity and adaptability that is required by these novel challenges to health research regulation. The choice of examples is illustrative of existing and novel research contexts where the concepts, tools, and mechanisms discussed in Part I come into play.

The first part of this section speaks to nascent challenges in the field of reproductive technologies, an area of health research often characterised by its disruptiveness to particular legal and social norms. The first two contributions to this theme focus on human gene editing, a field of research that erupted in global public ethical and policy debates when the live birth of twin girls, Lulu and Nana, whose genes had been edited in vitro, was announced by biophysician He Jiankui in 2018. For Isasi (Chapter 34), recent crises such as this provide opportunity to transform not only global policy on human germline gene editing, but collective behaviours in this field. In this chapter, she analyses the commonalities and divergences in international normative systems that regulate gene editing. For Isasi, a policy system that meaningfully engages global stakeholders can only be completely effective if we achieve both societal consensus and governance at local and global levels. For Chan (Chapter 35), the existence of multiple parallel discourses highlighted by Isasi can be used to facilitate broader representation of views within any policy solution. Chan considers the wider lessons that the regulatory challenges of human germline gene editing pose for the future of health research regulations. She posits that human germline gene editing is a "contemporary global regulatory experiment-in progress', which we can use to revisit current regulatory frameworks governing contentious science and innovation.

For the authors of the next two chapters, the order upon which existing regulatory approaches were built is being upended by new, dynamic sociotechnical developments that call into question the boundaries that law and regulation has traditionally relied upon. First, Hinterberger and Bea (Chapter 36) challenge us to consider how we might reconsider normative regulatory boundaries in their chapter on human animal chimeras - an area of biomedical research where our normative distinctions between human and animal are becoming more 
blurred as research advances. Here, the authors highlight the potential of interspecies research to perturb lasting, traditional regulatory models in the field of biomedical research. Next, McMillan (Chapter 37) examines the fourteen-day limit on embryo research as a current example of an existing regulatory tool - here a legal 'line in the sand' - that is being pushed to its scientific limits. She argues that recent advancements in in vitro embryo research challenge us to disrupt our existing legal framework governing the processual entity that is the embryo in vitro. For McMillan, disrupting our existing regulatory paradigms in embryo research enables essential policy discussion surrounding how we can, and whether we should, implement enduring regulatory frameworks in such a rapidly changing field.

For the second part of this section, the final two chapters examine the downstream effects of health research regulation in two distinct contexts. For these authors, it is clear that innovation in research practice and its applications requires us not only to disrupt our normative regulatory frameworks and systems, but to do so in a way that meaningfully engages stakeholders (see Laurie, Introduction). Jackson (Chapter 38) challenges the sufficiency of giving patients information about the limited evidence-base behind 'add-on' treatments that are available in fertility clinics, as a mechanism for safely controlling their use. For Jackson, regulation of these add-ons needs to go further; she argues that these treatments should be deemed by the Human Fertilisation and Embryology Authority - the regulator of fertility clinics and research centres in the UK - as 'unsuitable practices'. She highlights the combination of a poor evidence base for the success of these 'add-on' treatments and patients' understandable enthusiasm that these might improve fertility treatment outcomes. Her contribution confronts this 'perfect storm' of the uncertain yet potentially harmful nature of these add-ons, which are routinely 'oversold' in these clinics, yet under-researched. Jackson's offering gives us an example of an ongoing and increasing practice and process that requires us to disrupt prevailing regulatory norms. In the final chapter of this section, Harmon (Chapter 39) offers human enhancement as an example of how a regulatory regime, catalysed by disruptive research and innovation, has failed to capture key concepts. For Harmon, greater integration of humans and technology requires our regulatory frameworks to engage with 'identity' and 'integrity' more deeply, yet the current regulatory regime's failure to do so provides a lack of support and protection for human wellbeing.

Together, these chapters provide detailed analyses of carefully chosen examples and/or contexts that instantiate the necessity for reflexivity in a field where paradigms are (and should be) disturbed by health research and innovation. It is clear that particular regulatory feedback loops within and across particular regulatory spaces need to be closed in order to deliver authentic learning back to the system and to its users (see Laurie, Introduction and Afterword). A key theme in this section is the call to approach health research regulation as a dynamic endeavour, continually constituted by scientific processes and engaged with stakeholders and beneficiaries. In doing so, this section provides grounded assessments of HRR, showing the positive potential of responsive regulation as new approaches to health research attempt to meet the demands of an ever-changing world. 